\title{
Chronic cholecystitis with hilar bile duct stricture mimicking gallbladder carcinoma on positron emission tomography: A case report
}

\author{
QING-HONG KE, ZENG-LEI HE, XIN DUAN and SHU-SEN ZHENG \\ Department of Hepatobiliary and Pancreatic Surgery, The First Affiliated Hospital, College of Medicine, \\ Key Laboratory of Combined Multi-Organ Transplantation, Ministry of Health, \\ Zhejiang University, Hangzhou, Zhejiang 310003, P.R.China \\ Received December 1, 2012; Accepted February 5, 2013
}

DOI: $10.3892 / \mathrm{mco} .2013 .93$

\begin{abstract}
Thickening of the gallbladder wall is observed in patients with gallbladder carcinoma, as well as in those with chronic cholecystitis. It is difficult to distinguish between benign and malignant gallbladder wall thickening with conventional diagnostic imaging techniques, such as abdominal ultrasonography (US), computed tomography (CT) and magnetic resonance imaging (MRI), particularly in patients with bile duct strictures. Currently, the fluorine-18 2-fluorodeoxyglucose positron emission tomography/CT (F-18 FDG PET/ CT) scan is widely used in the differentiation of cholecystitis from gallbladder carcinoma. However, the F-18 FDG PET/CT scan may also be responsible for false-positive diagnosis. This case report focuses on a 74-year-old male who presented with thickening of the gallbladder wall and hilar bile duct stricture, originally misdiagnosed as gallbladder carcinoma by US and MRI. F-18 FDG PET/CT also demonstrated increased activity. This case was ultimately proven to be chronic cholecystitis by postoperative pathological examination and it is presented in order to emphasize the significance of considering the possibility of false-positive diagnosis by PET/CT, as a result of inflammatory lesions. Therefore, PET/CT should not be considered the gold standard for the discrimination between benign and malignant gallbladder wall thickening.
\end{abstract}

\section{Introduction}

Gallbladder carcinoma is the most common malignancy of the biliary system (1). The optimal treatment for gallbladder

Correspondence to: Dr Qing-Hong Ke, Department of Hepatobiliary and Pancreatic Surgery, The First Affiliated Hospital, College of Medicine, Key Laboratory of Combined Multi-Organ Transplantation, Ministry of Health, Zhejiang University, 79 Qingchun Road, Hangzhou, Zhejiang 310003, P.R.China E-mail: keqinghong@zju.edu.cn

Key words: thickened gallbladder wall, cholecystitis, positron emission tomography, false positive, gallbladder carcinoma carcinoma is radical resection. Thus, early detection is critical. However, thickening of the gallbladder wall is observed, not only in patients with gallbladder carcinoma, but also in those with chronic cholecystitis (2). It is difficult to distinguish between benign and malignant gallbladder wall thickening with conventional diagnostic imaging techniques, such as abdominal ultrasonography (US), computed tomography (CT) and magnetic resonance imaging (MRI), particularly in patients with bile duct strictures.

Currently, the fluorine-18 2-fluorodeoxyglucose positron emission tomography/CT (F-18 FDG PET/CT) scan is widely used in the differentiation between cholecystitis and gallbladder carcinoma and is also valuable for detection of regional lymph node involvement and unsuspected distant metastases (3). However, F-18 FDG PET/CT may lead to a false-positive diagnosis, particularly in cases of acute cholecystitis (4). We hereby present a case of thickening of the gallbladder wall with concurrent bile duct stricture, originally misdiagnosed as gallbladder carcinoma by US and MRI. F-18 FDG PET/CT also demonstrated increased activity. However, this case was ultimately proven to be chronic cholecystitis by postoperative pathological examination.

This study was approved by the Ethics Committee of Zhejiang University (Hangzhou, China). Written informed consent was obtained from the patient.

\section{Case report}

Patient. A 74-year-old man was referred to our hospital with signs of jaundice. On admission, he was free from abdominal pain and fever. The liver and spleen were not palpable and there was no sign of an abdominal mass. Murphy's sign was negative. In addition, the patient had been suffering from hypothyroidism for 15 years, following treatment with radioiodine (I-131) for hyperthyroidism, but had never received any thyroid hormone replacement medication.

Following admission, his alanine aminotransferase level was $36 \mathrm{U} / 1$ (normal range, 5-40 U/1), aspartate aminotransferase level $21 \mathrm{U} / 1$ (normal range, 8-40 U/l), $\gamma$-glutamyltransferase level $24 \mathrm{U} / 1$ (normal range, 15-80 U/l), alkaline phosphatase level $239 \mathrm{U} / 1$ (normal range, $<50 \mathrm{U} / \mathrm{l}$ ), total bilirubin level 
A

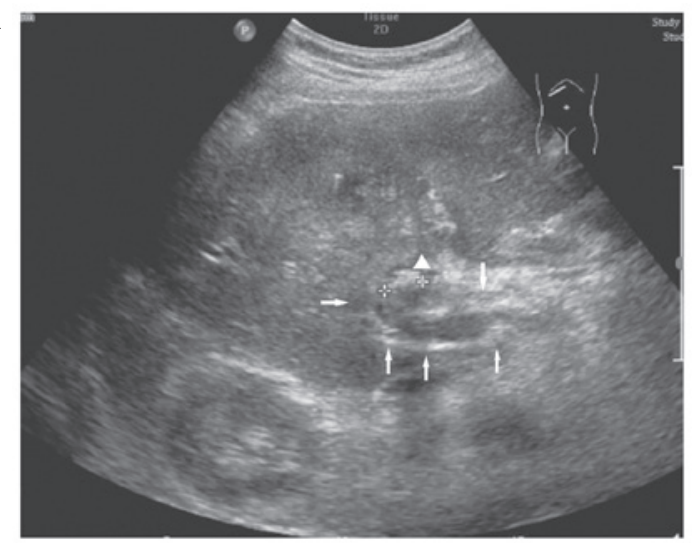

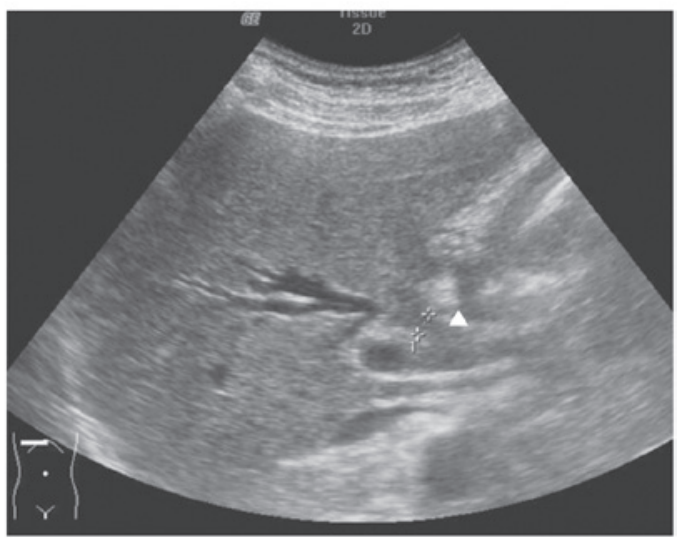

Figure 1. Ultrasonography demonstrated (A) thickened gallbladder wall (arrows) and intraluminal stone (arrowhead), with (B) perihilar bile duct stricture (arrowhead).
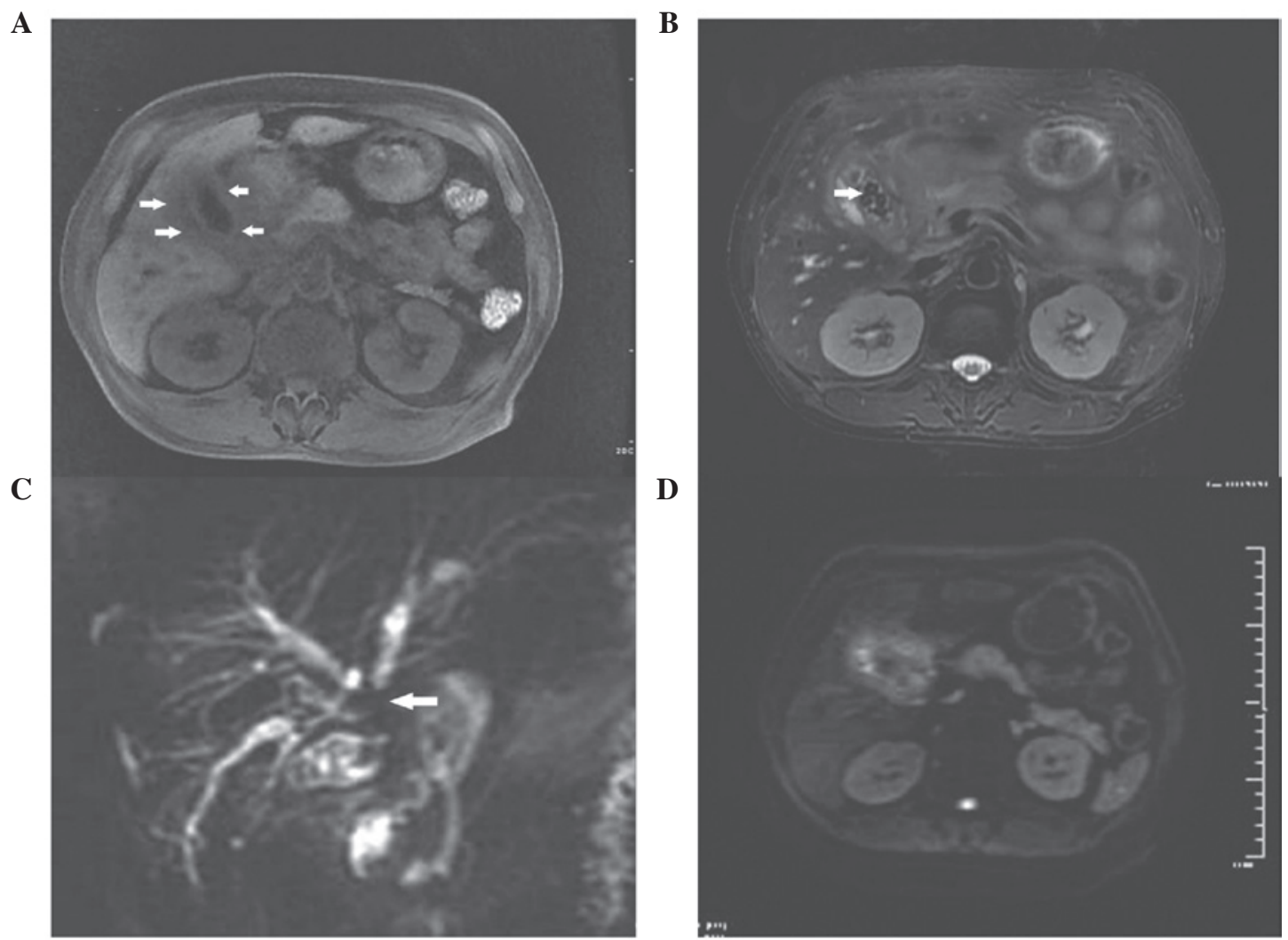

Figure 2. Magnetic resonance imaging (MRI) of the gallbladder. T1-weighted image demonstrated (A) thickened gallbladder wall (arrows) and (B) numerous gallstones (arrow). (C) Magnetic resonance (MR) cholangiography demonstrated hilar bile duct stricture (arrow) and dilation of the intrahepatic bile ducts. (D) Diffusion-weighted imaging demonstrated a positive signal in the gallbladder.

$56 \mu \mathrm{mol} / 1$ (normal range, 1.71-17.1 $\mu \mathrm{mol} / \mathrm{l}$ ) and direct bilirubin level $31 \mu \mathrm{mol} / 1$ (normal range, 1.71-7 $\mu \mathrm{mol} / \mathrm{l}$ ). Hepatitis $\mathrm{B}$ serology for HBsAg was negative and there was no hepatitis $\mathrm{C}$ infection or leucocytosis. The $\alpha$-fetoprotein and carbohydrate 19-9 levels were normal. C-reactive protein (CRP) level was $35 \mathrm{mg} / 1$ (normal, $<10 \mathrm{mg} / \mathrm{l}$ ). The patient had high thyroid-stimulating hormone (TSH) levels, with decreased levels of free T3 (FT3) and free T4 (FT4) (FT3=2.6 pmol/1, normal range 3.1-6.8 pmol/1; FT4=7.6 pmol/1, normal range $10.3-24.45 \mathrm{pmol} / 1$; and $\mathrm{TSH}=75.0 \mathrm{mIU} / 1$, normal range 0.4-4.0 mIU/1).
Findings. US demonstrated a thickened gallbladder wall and an intraluminal stone, as well as a perihilar bile duct stricture (Fig. 1). MRI demonstrated a thickened gallbladder wall and a positive signal in diffusion-weighted imaging (DWI). Magnetic resonance (MR) cholangiography demonstrated a hilar bile duct stricture and dilation of the intrahepatic bile ducts situated above the stricture (Fig. 2). F-18 FDG PET/CT also demonstrated increased activity in the gallbladder (Fig. 3), which also indicated the presence of gallbladder carcinoma. During the operation, a hard and thickened gallbladder wall was identified. Frozen section of the gallbladder was negative 


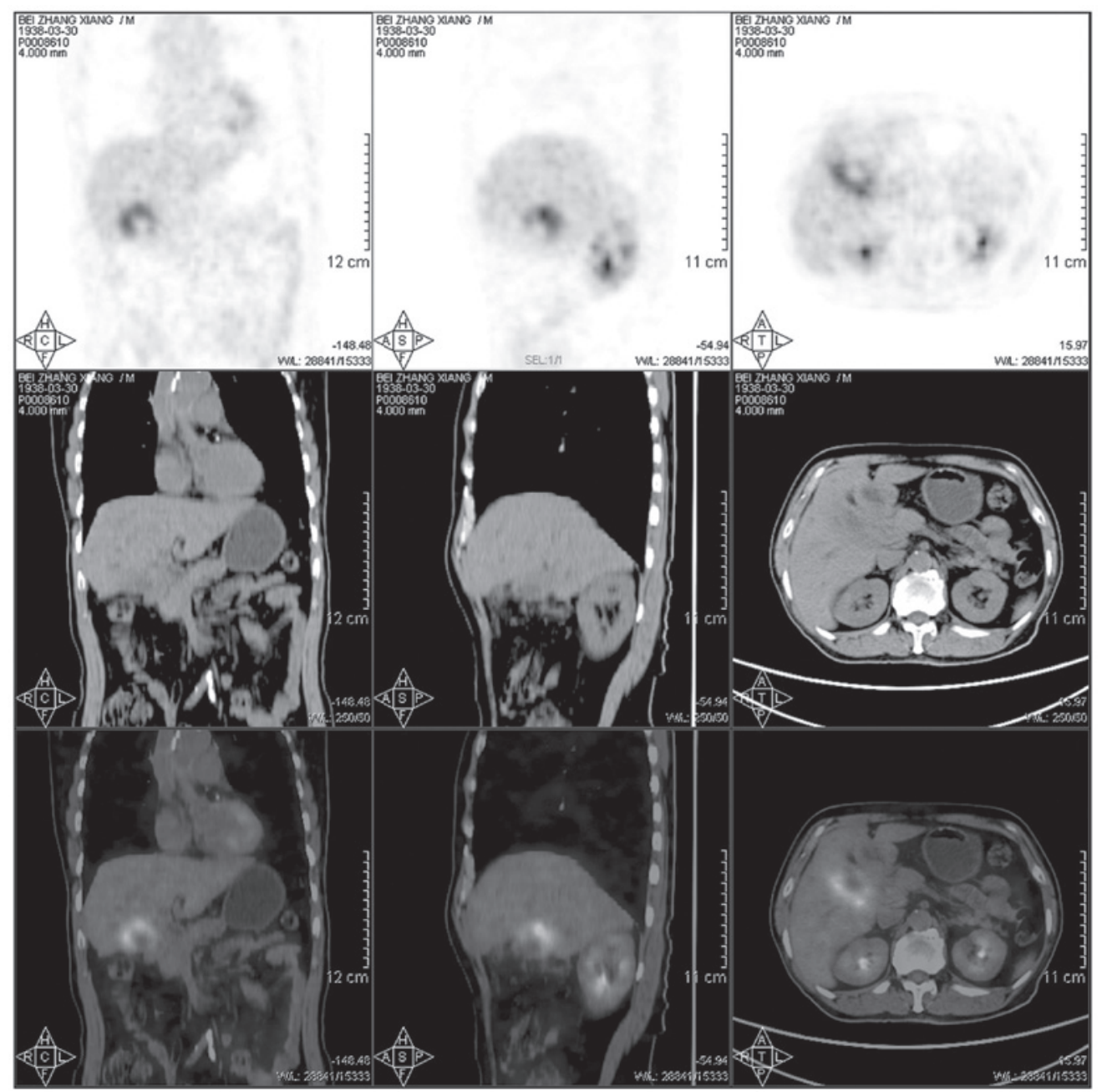

Figure 3. Fluorine-18 2-fluorodeoxyglucose positron emission tomography/computed tomography (F-18 FDG PET/CT) scan demonstrated intense F-18 FDG activity in the gallbladder fossa, which indicated gallbladder carcinoma.

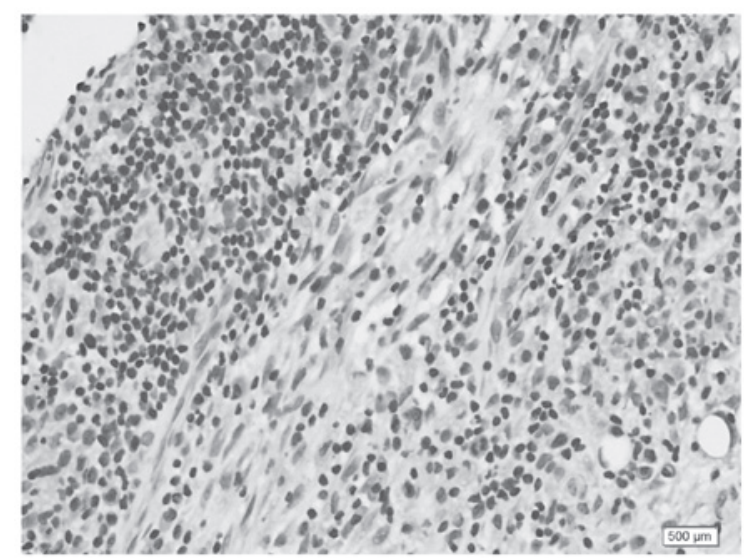

Figure 4. Frozen section of the gallbladder was negative for carcinoma. Final pathology (hematoxylin and eosin staining, magnification, $\mathrm{x} 400$ ) was consistent with chronic cholecystitis.

for carcinoma and revealed the presence of chronic cholecystitis (Fig. 4). The hilar bile duct stricture was also proven to be an inflammatory stricture. A T-tube was inserted to drain the bile, following bile duct exploration. Intra-operative blood loss was $100 \mathrm{ml}$. The patient recovered well after the operation and was discharged on the tenth post-operative day. The T-tube was removed two months after the operation and the patient was free from the jaundice.

\section{Discussion}

Thickening of the gallbladder wall has been considered an indication of gallbladder carcinoma or cholecysitis. However, this finding is non-specific and may represent a diagnostic challenge in the sense that its misinterpretation may result in delayed treatment.

CT and MRI scans play a critical role in the differentiation of cholecystitis from gallbladder carcinoma. However, the majority of imaging modalities, such as CT and MRI, are anatomical, which affects the accuracy of the diagnosis. By contrast, PET is a functional diagnostic imaging technique, that uses compounds labelled with F-18 FDG to measure cell metabolism. Therefore, F-18 FDG PET/CT exhibits significant advantages regarding the differentiation between benign and malignant lesions. However, F-18 FDG is not tumor-specific. This tracer may accumulate in inflammatory lesions as well, which results in low specificity in the differentiation of malignant tumors from benign lesions. Kitazono et al (4) reported their findings of ring-like radiotracer uptake in a patient 
diagnosed with acute cholecystitis. Xanthogranulomatous cholecystitis may also mimic gallbladder carcinoma, with a false-positive result on F-18 FDG PET/CT (5). Therefore, distinguishing benign from malignant gallbladder wall thickening is not always possible using F-18 FDG PET/CT.

In this case, the patient underwent DWI prior to the F-18 FDG PET/CT scan. Ogawa et al (6) reported that DWI may contribute to the improvement of the diagnostic ability for gallbladder wall thickening. This case demonstrated DWI positivity, which indicated gallbladder carcinoma. In addition to DWI positivity, we identified a hilar bile duct stricture. The majority of benign biliary strictures are attributed to iatrogenic injury or stones, although a few cases may be due to primary sclerosing cholangitis (7) or xanthogranulomatous choledochitis (8). The patient had no history of operation and MR cholangiopancreatography showed no multifocal strictures or dilated ducts, apart from the hilar stricture. The biliary stricture was first considered to be due to malignancy. All of the imaging findings favored the diagnosis of gallbladder cancer, which may affect the future diagnostic credibility of the F-18 FDG PET/CT scan.

During the diagnostic process, we also overlooked an important factor, which was the preoperative elevated CRP levels. The elevated CRP levels may also affect the accuracy of F-18 FDG PET/CT. Nishiyama et al (9) reported that the specificity of PET was higher in the group with normal CRP levels, compared to that in the group with elevated CRP levels. In this case, the patient had no fever, no abdominal pain and normal white blood cell count, which led us to mistakenly associate the elevated CRP level with malignancy. However, the postoperative pathological examination confirmed the diagnosis of chronic cholecystitis. We hypothesized that the relapsing cholecystitis induced the formation of fibrotic tissue around the gallbladder, which caused the bile duct stricture.
Due to the hypothyroidism, the patient had become insensitive to painful stimuli and only seeked medical advice after noticing the appearance of jaundice. Therefore, the recurrent inflammatory reaction in the gallbladder had been concealed.

PET/CT may be widely applied for the diagnosis of gallbladder disease. However, the possibility of false-positive results due to inflammatory lesions should be considered to avoid a misdiagnosis which may complicate further treatment.

\section{References}

1. Zhu AX, Hong TS, Hezel AF and Kooby DA: Current management of gallbladder carcinoma. Oncologist 15: 168-181, 2010.

2. Oe A, Kawabe J, Torii K, et al: Distinguishing benign from malignant gallbladder wall thickening using FDG-PET. Ann Nucl Med 20: 699-703, 2006.

3. Hansen N, Brown RK, Khan A, Frey KA and Orringer M: False positive diagnosis of metastatic esophageal carcinoma on positron emission tomography: a case report of cholecystitis simulating a hepatic lesion. Clin Nucl Med 35: 409-412, 2010.

4. Kitazono MT and Colletti PM: FDG PET imaging of acute cholecystitis. Clin Nucl Med 31: 23-24, 2006.

5. Makino I, Yamaguchi T, Sato N, Yasui $T$ and Kita I: Xanthogranulomatous cholecystitis mimicking gallbladder carcinoma with a false-positive result on fluorodeoxyglucose PET. World J Gastroenterol 15: 3691-3693, 2009.

6. Ogawa T, Horaguchi J, Fujita N, et al: High b-value diffusionweighted magnetic resonance imaging for gallbladder lesions: differentiation between benignity and malignancy. J Gastroenterol 47: 1352-1360, 2012

7. Helmberger H, Hellerhoff $K$, Rüll $\mathrm{T}$ and Rösch T: Chronic infections of the biliary system. Radiologe 40: 530-536, 2000.

8. Krishna RP, Kumar A, Singh RK, Sikora S, Saxena R and Kapoor VK: Xanthogranulomatous inflammatory strictures of extrahepatic biliary tract: presentation and surgical management. J Gastrointest Surg 12: 836-841, 2008.

9. Nishiyama Y, Yamamoto Y, Fukunaga K, et al: Dual-time-point 18F-FDG PET for the evaluation of gallbladder carcinoma. J Nucl Med 47: 633-638, 2006. 